POS PROCEEDINGS

\title{
Spectroscopy results from Belle
}

\section{Sookyung Choi (for the Belle Collaboration)*}

Gyeongsang National University

E-mail: schoi@gsnu.ac.kr

We report recent results on the charmonium and charmoniumlike states based on a large data sample recorded at the $\Upsilon(4 S)$ and $\Upsilon(5 S)$ resonances with the Belle detector at the KEKB asymmetricenergy $e^{+} e^{-}$collider.

The Xth Nicola Cabibbo International Conference on Heavy Quarks and Leptons, October 11-15, 2010

Frascati (Rome) Italy

${ }^{*}$ Speaker. 


\section{Introduction}

Starting with the observation of the $\eta_{c}(2 S)$ state, a number of new states have been observed by Belle. Some of these have been identified as being the predicted, but not-yet-seen, charmonium states, while others, designated by $X, Y \& Z$, are considered to be candidates for new types of charmoniumlike states such as hybrid $c \bar{c}$-gluon states or multiquark states either of the molecular type $(c \bar{q} \bar{c} q)$ or the diquark-diantiquark $(c q \overline{c q})$ type. Here, recent $X Y Z$ state-related measurements are reported and examined in the context of possible charmonium assignments.

\section{The $X(3872)$}

The $X(3872)$ was discovered by Belle in the $J / \psi \pi^{+} \pi^{-}$mass spectrum in exclusive $B \rightarrow$ $K X(3872)$ decay [1] in a $140 \mathrm{fb}^{-1}$ data sample; it was subsequently seen in three other experiments. One curious fact about the $X(3872)$ is the near equality of its mass and the $m_{D^{0}}+m_{D^{* 0}}$ threshold. The latest PDG world averaged mass [2] is $3871.56 \pm 0.22 \mathrm{MeV}$, while the $D^{0} D^{* 0}$ mass threshold is $3871.79 \pm 0.30 \mathrm{MeV}$. The CDF group reported that only the $J^{P C}$ options $1^{++}$and $2^{-+}$ are compatible with the $J / \psi$ and $\pi \pi$ helicity angle distributions [3]. It is also now well established that the $\pi \pi$ system in $X \rightarrow J / \psi \pi^{+} \pi^{-}$comes from $\rho \rightarrow \pi \pi$ decays.

\section{$2.1 X(3872) \rightarrow \gamma J / \psi\left(\psi^{\prime}\right)$}

The observation of $X(3872)$ in $\gamma J / \psi\left(\psi^{\prime}\right)$ final states ensures that the charge-conjugation parity of the $X(3872)$ is $C=+1$. The first reported evidence for $X(3872) \rightarrow \gamma J / \psi$ was given by Belle [4] using in $B \rightarrow K X(3872)$ decays in a $256 \mathrm{fb}^{-1}$ data sample with a signal significance of $\sim 4 \sigma$.

BaBar also reported $X(3872)$ decays to both $\gamma J / \psi$ and $\gamma \psi^{\prime}$ final states in the $B^{+} \rightarrow K^{+} \gamma J / \psi\left(\psi^{\prime}\right)$ process [5], with signal significances of $3.6 \sigma$ and $3.5 \sigma$, respectively. Their measured product of branching fractions are $B\left(B^{+} \rightarrow K^{+} X(3872)\right) \times B(X(3872) \rightarrow \gamma J / \psi)=(2.8 \pm 0.8) \times 10^{-6}$ and $B\left(B^{+} \rightarrow K^{+} X(3872)\right) \times B\left(X(3872) \rightarrow \gamma \psi^{\prime}\right)=(9.5 \pm 2.8) \times 10^{-6}$. The ratio of these branching fractions is $\frac{B\left(X_{3872} \rightarrow \gamma \psi^{\prime}\right)}{B\left(X_{3872} \rightarrow \gamma J \psi\right)}=3.4 \pm 1.4$, which is large for a $D \bar{D}^{* 0}$ molecular state [6]

This year Belle studied the $\gamma J / \psi\left(\psi^{\prime}\right)$ final states using their full data sample of $772 \times 10^{6} B \bar{B}$ events [7]. The $B \rightarrow K \gamma J / \psi$ channel is dominated by $B \rightarrow K \chi_{c 1} ; \chi_{c 1} \rightarrow \gamma J / \psi$ decays and this is used as a calibration reaction; the branching fraction for the well known $B^{+} \rightarrow K^{+} \chi_{c 1}$ decay is measured to be $(4.94 \pm 0.35) \times 10^{-4}$, which agrees well with the PDG value [2]. Belle also reported first evidence for $B \rightarrow K \chi_{c 2}$ (via $\chi_{c 2} \rightarrow \gamma J / \psi$ ) with $3.6 \sigma$ significance. The branching fraction for $B^{+} \rightarrow K^{+} \chi_{c 2}$ is measured to be $(1.11 \pm 0.37) \times 10^{-5}$. The ratio of branching fractions $\frac{\mathrm{B}\left(B^{+} \rightarrow K^{+} \chi_{c 2}\right)}{\mathrm{B}\left(B^{+} \rightarrow K^{+} \chi_{c 1}\right)}$ is $0.022 \pm 0.007$, which is a measure of the factorization suppression factor for $J^{P C}=2^{++}$. In the same $\gamma J / \psi$ final state but at higher masses, there is a clear $X(3872) \rightarrow \gamma J / \psi$ signal with $4.9 \sigma$ significance. Figure 1 shows $M_{\gamma J / \psi}$ mass distributions in exclusive (a) $B^{+} \rightarrow K^{+} \gamma J / \psi$ and (b) $B^{0} \rightarrow K_{S}^{0} \gamma J / \psi$ decay. The product of branching fractions for $B \rightarrow K X$ and $X \rightarrow \gamma J / \psi$ is measured to be $(1.8 \pm 0.5) \times 10^{-6}$, which agrees with the BaBar result. However, there is no significant signal for $B^{+} \rightarrow K^{+} X$ and $X \rightarrow \gamma \psi^{\prime}$ decay. Figures 1 show the $M_{\gamma \psi^{\prime}}$ mass distributions in $B^{+} \rightarrow K^{+} \gamma \psi^{\prime}$ decay for (c) $\psi^{\prime} \rightarrow \ell^{+} \ell^{-}$and (d) $\psi^{\prime} \rightarrow J / \psi \pi^{+} \pi^{-}$decays. An upper limit of the product of branching fractions is determined to be $B\left(B^{+} \rightarrow K^{+} X\right) \times\left(X \rightarrow \gamma \psi^{\prime}\right)<3.4 \times 10^{-6}$. The 

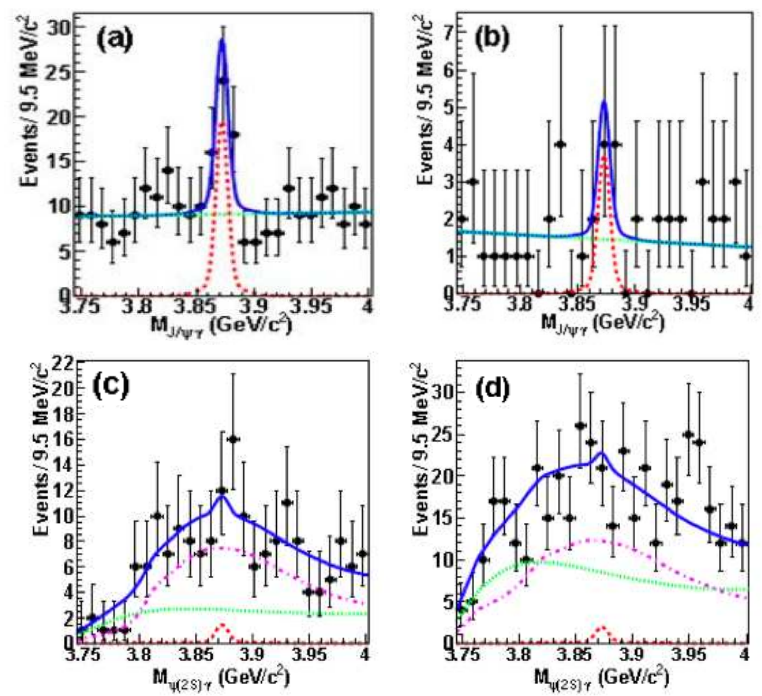

Figure 1: $\gamma J / \psi$ mass distributions (top) for (a) $B^{+} \rightarrow K^{+} X(3872)$ and (b) $B^{0} \rightarrow K_{s}^{0} X(3872)$ candidate events with subsequent $X(3872) \rightarrow \gamma J / \psi$ decay. $\gamma \psi^{\prime}$ mass distributions (bottom) for $B^{+} \rightarrow K^{+} X(3872)$ candidate events with subsequent $X(3872) \rightarrow \gamma \psi^{\prime}$ decay, where $\psi^{\prime} \rightarrow \ell^{+} \ell^{-}$in (c) and $\pi^{+} \pi^{-} J / \psi$ in (d).

ratio of branching fractions of its $90 \% \mathrm{CL}$ upper limit is $\frac{B\left(X \rightarrow \gamma \psi^{\prime}\right)}{B(X \rightarrow \gamma J / \psi)}<2.1$, in contradiction with the BaBar result.

\section{$2.2 X(3872) \rightarrow \omega J / \psi$}

In the $D^{0} \bar{D}^{* 0}$ molecular model of Swanson [6], the $J^{P C}$ is assumed to be $1^{++}$, in which case the $D^{0} \bar{D}^{* 0}$ component is dominant, with small admixtures of $\omega J / \psi$ and $\rho J / \psi$. In this model, $X(3872) \rightarrow \pi^{+} \pi^{-} \pi^{0} J / \psi$ decays were predicted to occur at about half the rate for $X(3872) \rightarrow$ $\pi^{+} \pi^{-} J / \psi$ decay. Belle performed a search for this $3 \pi J / \psi$ decay mode.

Figure 2(b) shows the $Y$ (3940) [8] seen in $\omega J / \psi$ mass distribution in $B \rightarrow K \omega J / \psi$ decay. In the $X(3872)$ mass region, which is at the right edge of the kinematic boundary, Belle observes a signal in the $3 \pi$ mass spectrum corresponding to the sub-threshold decay $X(3872) \rightarrow \omega J / \psi$. The measured number of signal events were $12.4 \pm 4.1$, from which the ratio of the branching fractions $\frac{B\left(X_{3872} \rightarrow \omega J / \psi\right)}{B\left(X_{3872} \rightarrow \pi^{+} \pi^{-} J / \psi\right)}$ is determined to be $1.0 \pm 0.5$ [4].

This year BaBar reanalyzed $B \rightarrow K \omega J / \psi$ final states using a relaxed omega mass selection [9] $0.5<m_{3 \pi}<0.9 \mathrm{GeV}$ and saw a similar $\omega$ signal. Using their reported branching fraction, we obtain the combined ratio from Belle and BaBar to be $\frac{B\left(X_{3872} \rightarrow \omega J / \psi\right)}{B\left(X_{3872} \rightarrow \pi^{+} \pi^{-} J / \psi\right)}=0.8 \pm 0.3$. In addition, BaBar reports that the $M_{3 \pi}$ mass spectrum from the $X_{3872} \rightarrow J / \psi \omega$ final states is suppressed near its upper kinematic boundary by a centrifugal barrier factor that is consistent with a $P$-wave. Their $P$-wave $\left(2^{-}\right)$fit $\left(\chi^{2} / \mathrm{NDF}=3.53 / 5\right)$ to the $M_{3 \pi}$ mass distribution is favored over their $S$-wave $\left(1^{+}\right)$ fit $\left(\chi^{2} / \mathrm{NDF}=10.17 / 5\right)$. This would be bad for a molecular interpretation of $X(3872)$, however, this corresponds to only about a $1.5 \sigma$ effect.

\subsection{Charmonium possibilities of $X(3872)$}

From the CDF angular analysis results [3], the only two possible $J^{P C}$ assignments for the $X$ 


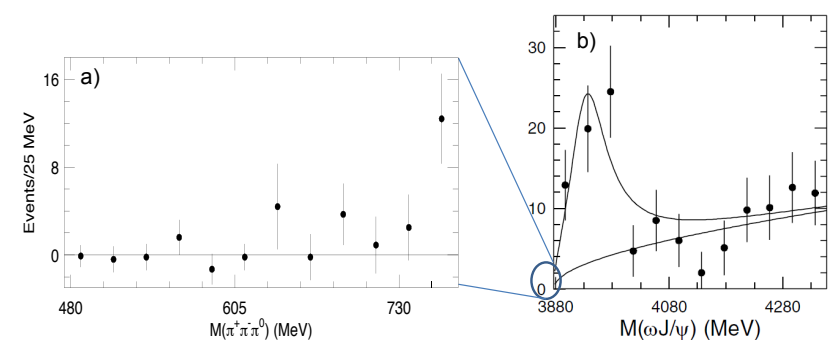

Figure 2: a) The B-meson signal yields versus $3 \pi$ invariant mass in the $X(3872)$ region of $M_{X}-3 \sigma<M_{X}<$ $\left.M_{X}+1 \sigma\right)$ for $B \rightarrow K \pi^{+} \pi^{-} \pi^{0} J / \psi$ decay. b) $B \rightarrow K \omega J / \psi$ signal yields versus $M(\omega J / \psi)$

are $1^{++}$and $2^{-+}$; all other $J^{P C}$ values are ruled out with high confidence. In this section, we survey charmonium possibilities for $X(3872)$ with these two $J^{P C}$ assignments.

For the $1^{++}$assignment, the possible undiscovered charmonium state is $\chi_{c 1}^{\prime}$. For the $X(3872)=$ $\chi_{c 1}^{\prime}$ assignment, the following puzzling questions arise.

- Since the mass of $\chi_{c 2}^{\prime}$ is now known to be $3930 \mathrm{MeV}$ [10], the mass of $\chi_{c 1}^{\prime}$ is expected to be $\sim 3905 \mathrm{MeV}$. Therefore, the mass of $X(3872)$ is too low for it to be the $\chi_{c 1}^{\prime}$.

- Barnes et al. [11] estimated the partial width for $\Gamma\left(\chi_{c 1} \prime \rightarrow \gamma \psi^{\prime}\right) \sim 180 \mathrm{keV}$, while $\Gamma\left(\chi_{c 1} \prime \rightarrow\right.$ $\gamma J / \psi) \sim 14 \mathrm{keV}$. So, the ratio of of partial widths $\frac{\Gamma\left(\chi_{c 1} \rightarrow \gamma \psi^{\prime}\right)}{\Gamma\left(\chi_{c 1} / \rightarrow \gamma J / \psi\right)}$ should be much bigger than unity. Therefore, the $\chi_{c 1}$ ' assignment would be a possible option if the BaBar measurement is right, which gives a large partial width for the $\gamma \psi^{\prime}$ mode.

- $\Gamma\left(X \rightarrow \pi^{+} \pi^{-} J / \psi\right)=(3.4 \pm 1.2) \times \Gamma(X \rightarrow \gamma J / \psi)$ is estimated to be about $45 \mathrm{keV}$ using the Barnes value for $\Gamma_{\gamma J / \psi}$ and the measured ratio of $\frac{\Gamma_{\pi \pi J / \psi}}{\Gamma_{\gamma J / \psi}}$. This partial width for $X \rightarrow$ $\pi^{+} \pi^{-} J / \psi$ decay is huge for an isospin-violating transition; other isospin violating transitions in the charmonium system are less than $1 \mathrm{keV}\left(\right.$ e.g., $\left.\Gamma\left(\psi^{\prime} \rightarrow \pi^{0} J / \psi\right) \sim 0.4 \mathrm{keV}[2]\right)$.

For the $2^{-+}$assignment, the possible undiscovered charmonium state is the singlet $D$-wave state known as the $\eta_{c 2}\left({ }^{1} D_{2}\right)$. For this assignment, the following questions arise.

- Y. Jia et al. [12] estimated the partial widths to be $\Gamma\left(\eta_{c 2} \rightarrow \gamma \psi^{\prime}\right) \sim 0.4 \mathrm{keV}$ and $\Gamma\left(\eta_{c 2} \rightarrow\right.$ $\gamma J / \psi) \sim 9 \mathrm{keV}$. This favors the Belle measurement where the ratio $\frac{\Gamma\left(\eta_{c 2} \rightarrow \gamma \psi^{\prime}\right)}{\Gamma\left(\eta_{c 2} \rightarrow \gamma J / \psi\right)}$ is smaller.

- Using the well established mass of its multiplet partner, $M\left(\psi^{\prime \prime}\right)=3770 \mathrm{MeV}$, the mass of the $\eta_{c 2}$ is estimated to be $3837 \mathrm{MeV}$. Thus, the $X(3872)$ mass is high for $\eta_{c 2}$.

- $\Gamma\left(X \rightarrow \pi^{+} \pi^{-} J / \psi\right)=(3.4 \pm 1.2)(X \rightarrow \gamma J / \psi)$ is about $30 \mathrm{keV}$ using the Jia $\gamma J / \psi$ width. This is also large for an isospin-violating transition.

- For the $2^{-+}\left(\eta_{c 2}\right)$ assignment, the branching fraction for the $B^{+} \rightarrow K^{+} \eta_{c 2}$ is too high for a non-factorizable decay. Other two $B^{+} \rightarrow K^{+} h_{c}$ and $B^{+} \rightarrow K^{+} \chi_{c 2}$ decay, which is also nonfactorizable and suppressed by an angular momentum barrier, are just barely seen in the huge Belle data sample.

- The branching fraction for $\eta_{c 2} \rightarrow D \bar{D}^{*}$ is expected to be small [13], but the averaged ratio from both Belle and BaBar is $\frac{\Gamma(X \rightarrow D D *)}{\Gamma(X \rightarrow \pi \pi J / \psi)}=9.5 \pm 3.1$, which is high for the $\eta_{c 2}$. 


\section{More $X$ and $Y$ states near $3940 \mathrm{MeV}$}

Belle observed three states near $3940 \mathrm{MeV}$ via three different production and decay channels $[8,10,14]$. Among these three, the $Z(3930)$ state, which is produced in the $\gamma \gamma \rightarrow D \bar{D}$ process, is generally considered to be the charmonium $\chi_{c 2}^{\prime}$ state, even though the mass $M=3929 \pm 5 \pm 2 \mathrm{MeV}$ is somewhat lower than potential model predictions. The $X(3940)$ is observed in the $D \bar{D}^{*}$ mass spectrum from double charmonium production in $e^{+} e^{-} \rightarrow J / \psi D^{*} \bar{D}$ annihilation and the $Y(3940)$ is observed in the $\omega J / \psi$ mass spectrum in $B \rightarrow K \omega J / \psi$ decays. The mass and width of the $X(3940)(Y(3940))$ are measured to be $\mathrm{M}=3942_{-6}^{+7} \pm 6(3943 \pm 11 \pm 13) \mathrm{MeV}$ and $\Gamma=37_{-15}^{+26} \pm 8$ $(87 \pm 22 \pm 26) \mathrm{MeV}$. Although the masses are similar, the $X(3940)$ and $Y(3940)$ appear to be different states: the $X(3940)(Y(3940))$ has not been seen in the $\omega J / \psi\left(D \bar{D}^{*}\right)$ final state in $B \rightarrow X(Y) K$ decays.

It is important to search for $\omega J / \psi\left(\right.$ or $D \bar{D}^{*}$ ) in two-photon collisions, where its spin-parity of resonance is preferentially constrained to be $J^{p}=0^{ \pm}$or $2^{ \pm}$. Belle observed a $7.7 \sigma$ enhancement in the $\omega J / \psi$ system [15] produced in the $\gamma \gamma \rightarrow \omega J / \psi$ process; the mass and total width are measured to be $\mathrm{M}=3915 \pm 3 \pm 2 \mathrm{MeV}$ and $\Gamma=17 \pm 10 \pm 3 \mathrm{MeV}$. This state, denoted by $X(3915)$, is probably related to one of the three above-mentioned states in the 3.90-3.95 GeV mass region. If we assume the $X(3915)$ is $0^{+}\left(2^{+}\right)$resonance, the product of the two-photon decay width and branching fraction to $\omega J / \psi$ is determined to be $\Gamma_{\gamma \gamma}(X(3915)) \mathrm{B}(\mathrm{X} \rightarrow \omega \mathrm{J} / \psi)=61 \pm 17 \pm 8(18 \pm 5 \pm 2) \mathrm{eV}$ for $J^{p}=0^{+}\left(2^{+}\right)$. For comparison, the measured product of the two-photon decay width and branching fraction for $Z(3930) \rightarrow D \bar{D}$ is $180 \pm 50 \pm 30 \mathrm{eV}$. If the $X(3915)$ is the $Z(3930)\left(\chi_{c 2}^{\prime}\right)$, the ratio of branching fractions $\frac{\mathrm{BF}\left(\chi_{\mathrm{c} 2}^{\prime} \rightarrow \omega \mathrm{J} / \psi\right)}{\mathrm{BF}\left(\chi_{\mathrm{c} 2}^{\prime} \rightarrow \mathrm{D} \overline{\mathrm{D}}\right)}$ is large for an above-open-charm-threshhold charmonium state. Also, for both the $0^{+}$and $2^{+}$options, if we assume that the $\gamma \gamma$ partial width is $\sim 1 \mathrm{keV}$, which is typical for charmonium states, $\Gamma_{\omega J} / \psi$ would be of the order of $1 \mathrm{MeV}$, which is large for charmonium.

The $\Gamma\left(Y_{3940} \rightarrow \omega J / \psi\right)$ partial width is also estimated to be large using the averaged product branching fraction from Belle and BaBar to be $B\left(B^{+} \rightarrow K^{+} Y_{3940}\right) \times B\left(Y_{3940} \rightarrow \omega J / \psi\right)=$ $(5.0 \pm 0.8) \times 10^{-5}$ and the PDG averaged $\Gamma\left(Y_{3940}\right)=40_{-13}^{+18} \mathrm{MeV}$. If we assume the maximum possible branching fraction for $B \rightarrow K Y_{3940}$ is $10 \times 10^{-4}$ (the branching fraction for $B \rightarrow K J / \psi$ ), the partial width for $\Gamma\left(Y_{3940} \rightarrow \omega J / \psi\right)$ is determined to be larger than order $1 \mathrm{MeV}$, which is large for conventional charmonium.

\section{The $Y(4260)$ and $Y_{b}$}

The $1^{--} Y(4260)$ state was first discovered by BaBar [16] and confirmed by Belle [17] in the $J / \psi \pi^{+} \pi^{-}$in radiative $e^{+} e^{-} \rightarrow \gamma_{I S R} Y(4260)$ process. The partial width for $Y \rightarrow \pi^{+} \pi^{-} J / \psi$ is determined to be larger than $0.5 \mathrm{MeV}$ at the $90 \% \mathrm{CL}$ level by Mo et al. [18], which is much larger than that for $\psi^{\prime} \rightarrow \pi^{+} \pi^{-} J / \psi$. This large partial width is one of the remarkable properties of the $Y(4260)$ that have led to various exotic interpretations of its quark content. An interesting question is whether or not there exist counterparts in the $s \bar{s}$ and/or $b \bar{b}$ quark systems.

Belle reported an anomalously large $e^{+} e^{-} \rightarrow \Upsilon(1,2 S) \pi^{+} \pi^{-}$production cross section near the peak of the $\Upsilon(5 S)$ resonance at $\sqrt{s} \sim 10.87 \mathrm{GeV}$ measured with a $21.7 \mathrm{fb}^{-1}$ data sample [19]. If they assume that the signal events only come from decays of the $\Upsilon(5 S)$ resonance, their extracted partial 


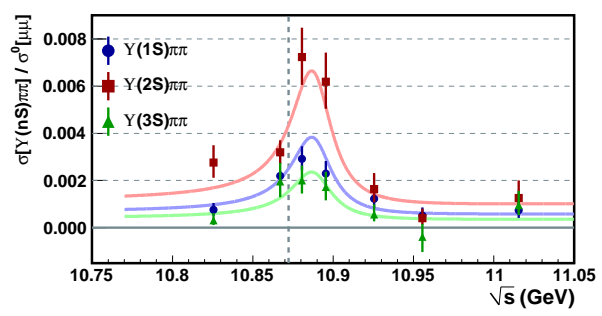

Figure 3: The $\Upsilon(n S) \pi \pi$ (n=1,2 and 3) cross sections near the $\Upsilon(5 S)$ resonance show peak structure that deviates from the peak (vertical line) of $\Upsilon(5 S)$ obtained from the hadronic cross sections.

widths are $\sim 300$ times larger than those for corresponding transitions from the $\Upsilon(4 S)$. Recently, Belle measured the energy dependence of the $e^{+} e^{-} \rightarrow \Upsilon(n S) \pi \pi(\mathrm{n}=1,2,3)$ production cross section using data accumulated at seven different $\mathrm{cm}$ energy points near the $\Upsilon(5 S)$ resonance. A new common peak structure was observed for all three $e^{+} e^{-} \rightarrow \Upsilon(1,2,3 S) \pi^{+} \pi^{-}$cross sections. A fit using Breit-Wigner resonance function with a common mass and width to these peaks, shown in Fig. 3, gives a mass and width of $\mathrm{M}=10888.4_{-2.6}^{+2.7} \pm 1.2 \mathrm{MeV}$ and $\Gamma=30.7_{-7.0}^{+8.3} \pm 3.1 \mathrm{MeV}[20]$, which are not consistent with any known $b \bar{b}$ state such as the $\Upsilon(10860)$. This can be considered to be a candidate for a $Y_{b}$-type state in the $b \bar{b}$ system.

\section{Charged $Z^{+}$states}

A charged charmonium-like state could not be a $c \bar{c}$ charmonium state; its minimal quark structure would have to be a $c \bar{c} u \bar{d}$ tetraquark arrangement. The charged $Z(4430)^{+}$state was first observed by Belle as a peak in the $\pi^{+} \psi^{\prime}$ mass distribution in exclusive $B \rightarrow K \pi^{+} \psi^{\prime}$ decays[21]. It was confirmed by a subsequent reanalysis using a Dalitz plot formalism [22] that includes all possible intermediate $K \pi$ resonances. This Dalitz analysis method was first employed in the observation of two other charged $Z_{1}^{+}$and $Z_{2}^{+}$states that are seen to decay to $\pi^{+} \chi_{c 1}$ final states in exclusive $B \rightarrow K \pi^{+} \chi_{c 1}$ decays [23]. The Dalitz-plot analysis demonstrated that these $Z$ states are not produced by reflections from any known and possibly unknown resonances in the $K \pi$ channel. However, BaBar searched for the $Z(4430)^{+}$, but did not see a significant signal [24].

\section{Acknowledgments}

This work was supported by the Korea Research Foundation Grant funded by the Korean Government (KRF-2008-313-C00177).

\section{References}

[1] S.-K. Choi, S.L. Olsen et al. (Belle Collaboration), Observation of a narrow charmoniumlike state in exclusive $B^{ \pm} \rightarrow K^{ \pm} \pi^{+} \pi^{-} J / \psi$ decay, Phys. Rev. Lett. 91, 262001 (2003).

[2] 2010 Updated Table from Particle Data Group

[3] A. Abulencia et al. (CDF Collaboration), Analysis of the Quantum Numbers $J^{P C}$ of the X(3872) Particle, Phys. Rev. Lett. 98, 132002 (2007). 
[4] K.Abe et al. (Belle Collaboration), Evidence for $X(3872) \rightarrow \gamma J / \psi$ and the sub-threshold decay $X(3872) \rightarrow \omega J / \psi,[$ arXiv: hep-ex/0505037] (2005).

[5] B. Aubert et al. (BaBar Collaboration), Evidence for $X(3872) \rightarrow \psi(2 S) \gamma$ in $B^{ \pm} \rightarrow X(3872) K^{ \pm}$ decays, and a study of $B \rightarrow c \bar{c} \gamma K$, Phys. Rev. Lett 102, 132001 (2009).

[6] E.S.Swanson, Diagonostic decays of the X(3872), Phys. Lett. B598, 197 (2004).

[7] V. Bhardwaj, talk at the International Workshop on Heavy Quarkonia(QWG7 at Fermilab(2010).

[8] S.-K. Choi, S.L. Olsen et al. (Belle Collaboration), Observation of a near-threshold $\omega J / \psi$ mass enhancement in exclusive $B \rightarrow K \omega J / \psi$ decay, Phys. Rev. Lett. 94, 182002 (2005).

[9] P.del Amo Sanchez et al. (BaBar collaboration), Evidence for the decay $X(3872) \rightarrow J / \psi \omega$, Phys. Rev. D82, 011001 (2010).

[10] S. Uehara et al. (Belle Collaboration), Observation of a $\chi_{c 2}^{\prime}$ Candidate in $\gamma \gamma \rightarrow D \bar{D}$ Production at Belle, Phys. Rev. Lett. 96, 082003 (2006).

[11] T. Barnes, S. Godfrey and E.S. Swanson, Higher Charmonia, Phys. Rev. D72, 054026 (2005).

[12] Y. Jia, W. Sang and J. Xu, Is the $J^{P}=2^{-}$assignment for the $X(3872)$ compatible with the radiative transition data?, [arXiv:1007.4541v2 [hep-ph]].

[13] Y.S. Kalashnikova and A.V. Nefediev, X(3872) as $a^{1} D_{2}$ charmonium state, [arXiv:1008.2895v2 [hep-ph]].

[14] P. Pakhlov et al. (Belle Collaboration), Production of new charmoniumlike states in $e^{+} e^{-} \rightarrow J / \psi D^{*} \bar{D}^{*}$ at $\sqrt{s} \sim 10.6 \mathrm{GeV}$, Phys. Rev. Lett. 100, 202001 (2008).

[15] S. Uehara, et al. (Belle Collaboration), Observation of a charmonium-like enhancement in the $\gamma \gamma \rightarrow \omega J / \psi$ process, Phys. Rev. Lett. 104, 092001 (2010).

[16] B. Aubert et al. (BaBar Collaboration), Observation of a Broad Structure in the $\pi^{+} \pi^{-} J / \psi$ Mass spectrum at 4.26GeV/c², Phys. Rev. Lett. 95, 142001 (2006).

[17] C.Z. Yuan et al. (Belle Collaboration), Measurement of the $e^{+} e^{-} \rightarrow \pi^{+} \pi^{-} J / \psi$ Cross section via Inotial state Radiation at Belle, Phys. Rev. Lett. 99, 182004 (2007).

[18] X.H.Mo et al. Determining the upper limit of $\Gamma_{e e}$ for the $Y(4260)$, Phys. Lett. B 640, 182 (2006).

[19] K.F.Chen et al. (Belle Collaboration), Observation of Anomalous $\Upsilon(1 S) \pi^{+} \pi^{-}$and $\Upsilon(2 S) \pi^{+} \pi^{-}$ Production near rhe $\Upsilon(5 S)$ Resonance, Phys. Rev. Lett. 100, 112001 (2008).

[20] K.F.Chen et al. (Belle Collaboration), Observation of an enhancement in $e^{+} e^{-}$to $\Upsilon(1 S) \pi^{+} \pi^{-}, \Upsilon(2 S) \pi^{+} \pi^{-}$, and $\Upsilon(3 S) \pi^{+} \pi^{-}$production near $\sqrt{s}=10.89 \mathrm{GeV}$ at Belle, Phys. Rev. $D$ 82, 091106 (2010).

[21] S.-K. Choi, S. L. Olsen et al. (Belle Collaboration), Observation of a resonance-like structure in the $\pi^{ \pm} \psi^{\prime}$ mass distribution in exclusive $B \rightarrow K \pi^{ \pm} \psi^{\prime}$ decays, Phys. Rev. Lett. 100, 142001 (2008).

[22] R. Mizuk et al. (Belle Collaboration), Dalitz analysis of $B \rightarrow K \pi^{+} \psi^{\prime}$ decays and the $Z(4430)^{+}$, Phys. Rev. D80, 031104 (2009).

[23] R. Mizuk, R. Chistov et al. (Belle Collaboration), Observaion of two resonance-like structures in the $\pi^{+} \chi_{c 1}$ mass distribution in exclusive $\bar{B}^{0} \rightarrow K^{-} \pi^{+} \chi_{c 1}$ decays, Phys. Rev. D 78, 072004 (2008).

[24] B. Aubert et al. (BaBar Collaboration), Search for the $Z(4430)^{+}$at BABAR, Phys. Rev. D79, 112001 (2009). 